\title{
PLASTICITY IN THE SPINAL CORD SENSORY MAP FOLLOWING PERIPHERAL NERVE INJURY IN RATS'
}

\author{
MARSHALL DEVOR*,2 AND PATRICK D. WALL \\ * Life Sciences Institute, Hebrew University of Jerusalem, Jerusalem 91904, Israel and $\ddagger$ Cerebral Functions Research Group, Department of \\ Anatomy and Embryology, University College London, London, WC1E 6BT, England
}

\begin{abstract}
The medial part of the L4 and 5 dorsal horn in adult rats is dominated by afferents from the toes and foot. After transection of the sciatic and saphenous nerves, virtually all cells in this region are left without any peripheral receptive field. Beginning 4 to 5 days after nerve section, however, many peripherally deafferented cells take on a novel receptive field on the thigh, lower back, or perineum. The new receptive fields are served by intact nerves ending in proximal skin rather than by misdirected sprouts of cut toe-foot nerves. Thus, peripheral axotomy results in synaptic reorganization in the spinal cord proper. Receptive field reorganization occurs after nerve transection, ligation, or ligation with distal transection but does not occur if the nerve is crushed. If a cut nerve is sutured and regeneration is permitted, spinal reorganization is reversed and the toe-foot afferents regain exclusive dominance of the medial dorsal horn.
\end{abstract}

The skin surface is mapped in an orderly fashion onto second order sensory neurons in the dorsal horn (e.g., Brown and Fuchs, 1975; Devor and Claman, 1980). For example, within the lumbar enlargement, receptive fields of cells in the medial part of the dorsal horn are restricted to the toes and foot; cells in the lateral part of the dorsal horn have receptive fields on the thigh, lower back, or perineum (Fig. 1). As is to be expected, if one cuts the peripheral nerves that serve the foot (sciatic and saphenous nerves), virtually all cells in the medial dorsal horn are left without a receptive field of any sort. We showed recently in cats, however, that within a few weeks after nerve section, the peripherally deafferented cells acquire a novel receptive field on the thigh, lower back, or perineum (Devor and Wall, 1978, 1981). This phenomenon differs in an important respect from the plastic changes that occur following transection of dorsal roots (Liu and Chambers, 1958; Illis, 1973; Goldberger and Murray, 1974; Basbaum and Wall, 1976). After dorsal rhizotomy, axon terminal branches within the spinal cord are separated from the cell soma and therefore undergo rapid Wallerian degeneration. Neighboring uncut afferents then are thought to sprout new processes. Here, because the injury is distal to the dorsal root ganglion, central synaptic terminals remain in continuity with the cell soma. Although subtle retrograde changes do occur in the central terminals of peripherally axotomized fibers, they do not

\footnotetext{
${ }^{1}$ This work was supported by the Fritz Thyssen Stiftung and the C H. Revson Foundation. We wish to thank I. Frank and R. GovrinLippmann for their assistance.

${ }^{2}$ To whom correspondence should be addressed.
}

undergo massive degeneration and, in fact, synaptic transmission remains intact for some time (Wall and Devor, 1981; Wall et al., 1981). So far, there is no evidence that thigh or perineal afferents sprout in the spinal cord after toe-foot nerves have been cut (Devor and Claman, 1980).

Synaptic rearrangement in cats was first detected about 1 week after nerve section, and it did not stabilize until the end of the 4th postoperative week (Devor and Wall, 1981). This lag time poses some difficulties for a further analysis of the mechanisms involved. We, therefore, repeated our experiments in rats and found that the plastic changes seen in cats also occur in rats only much more quickly. Furthermore, we found that the rearrangement of dorsal horn somatotopy is reversible upon nerve regeneration and that it occurs if the peripheral nerve is cut but does not occur if the nerve is crushed.

\section{Materials and Methods}

Experiments were performed on adult (250- to 350-gm) male rats of the Wistar-derived Sabra strain. The rats were studied acutely or at various intervals after nerve injury. For chronic experiments, the rats were anesthetized with ether, and the sciatic and saphenous nerves were exposed unilaterally. In 20 rats, the saphenous nerve was ligated and cut distally, and the sciatic nerve was either ligated and cut $(n=14)$, ligated but not cut ( $n=$ $2)$, or cut but not ligated $(n=4)$. In cases where the nerve was cut, about 3 to $5 \mathrm{~mm}$ of the distal stump was excised. In 11 rats, the saphenous nerve was ligated and cut, and the sciatic nerve was crushed for $30 \mathrm{sec}$ with a smooth jawed hemostat (jaw width, $2.5 \mathrm{~mm}$ ). The posi- 


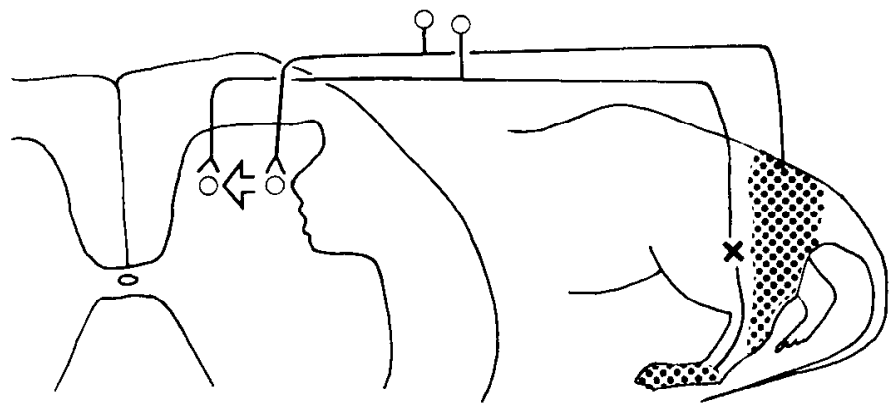

Figure 1. Schematic representation of the distribution of the nerves of the thes and foot (sciatic and saphenous nerves) and the nerves of the posterior thigh (posterior cutaneous thigh, perineal, and pudendal nerves) in the hindlimb and in the lower lumbar spinal cord in rats. Transection of the toe-foot nerves in the periphery $(X)$ causes medial dorsal horn cells to acquire novel receptive fields on the thigh.

tion of the crush was marked with carbon. We have determined previously that such a crush lesion totally blocks nerve conduction and causes Wallerian degeneration of all fibers in the distal stump (Devor et al., 1979). Finally, in 3 rats, the sciatic nerve was cut and its ends were re-approximated immediately with 10-0 sutures. In 2 of the 3 , the suture line was covered with a Silastic cuff. Following the nerve procedure, the wound was closed, 20,000 units of penicillin was administered intramuscularly, and the rats were allowed to recover. All three types of nerve injury rendered the toes and foot completely anesthetic.

After 2 to 147 days, the rats were studied in acute electrophysiological preparations performed under pentobarbital anesthesia $(50 \mathrm{mg} / \mathrm{kg}$, then $20 \mathrm{mg} / \mathrm{kg} / \mathrm{hr})$. In all cases, the sciatic and saphenous nerves on the experimental side were (re)sectioned 5 to $10 \mathrm{~mm}$ proximal to the original cut or crush, the sciatic and saphenous nerves on the previously intact side were cut acutely, and then the lumbosacral spinal cord was exposed and placed under warmed $\left(37^{\circ} \mathrm{C}\right)$ mineral oil. In most experiments, the animals were paralyzed with Flaxedil and artificially respired through a tracheal cannula. Arterial blood pressure (carotid), heart rate, temperature $\left(37^{\circ} \mathrm{C}\right)$, and expired $\mathrm{PCO}_{2}$ were monitored and maintained.

The experiments began with a series of closely spaced electrode penetrations on the acutely cut side heginning in the dorsal columns and moving laterally. We used $3 \mathrm{M}$ $\mathrm{KCl}$-filled 1- to 5-megohm micropipettes. The stimulus procedure was to search for receptive fields on the skin of the entire ipsilateral hindquarter. Stimuli were hair movement, touch (up to about $100 \mathrm{gm} / \mathrm{cm}^{2}$ ) with a blunt probe, pressure (over $100 \mathrm{gm} / \mathrm{cm}^{2}$ ), and pinch using rattoothed forceps. Since we explored the skin continuously, the sample of cells studied was not restricted to those with spontaneous discharge. The first track in which at least 2 cells could be detected within $500 \mu \mathrm{m}$ of the cord surface was marked by cutting off the electrode in situ after it had penetrated $1500 \mu \mathrm{m}$. Histological verification using thick $(0.5-$ to $1.0-\mathrm{mm}$ ) cleared sections (Wall and Werman, 1976) later showed that these marker electrodes consistently ran along the medial border of the dorsal horn. Penetrations then were continued laterally in the steps of 50 to $100 \mu \mathrm{m}$ until the first position in which at least 2 cells were encountered that had cutaneous receptive fields (of any sort) within $1000 \mu \mathrm{m}$ of the cord surface was reached. The tip of this electrode too was cut off as a marker (see Fig. 2). Mapping then proceeded on the side of the chronic nerve lesion. First, the medial edge of the dorsal horn was located and marked as on the control side. Then, a second marker electrode was placed lateral to the first by a distance equal to the distance between the two marker electrode tips on the control side. Cells in the zone between the two markers on the experimental and control sides, that is, within the toe-foot map in medial part of the dorsal horn, were the main object of our study (Fig. 2). About five evenly spaced penetrations were made through the medial dorsal horn on each side.

\section{Results}

Intact rats. As in cats, the medial part of the dorsal horn in intact rats is occupied almost exclusively by cells with excitatory receptive fields restricted to the ipsilateral toe and foot. Starting in the dorsal column and moving laterally, cells with receptive fields on skin proximal to the ankle were not encountered until the search electrode was moved more than $400 \mu \mathrm{m}$ lateral to the medial edge of the grey matter (Fig. 2). We were able to isolate about 5 cells with toe-foot receptive fields per penetration to $1000 \mu \mathrm{m}$ in the medial region in intact rats. This figure should be taken as a lower limit, however, as it was frequently impossible to obtain an exact cell count in a crowded field. Measured at a depth of 500 $\mu \mathrm{m}$, the total width of the dorsal horn grey matter in our rats was about $650 \mu \mathrm{m}$. Thus, the medial $60 \%$ or so of the rat L4 and 5 dorsal horn is devoted exclusively to the toes and foot.

Acute nerve section. In 51 electrode penetrations to $1000 \mu \mathrm{m}$ in 13 acutely neurectomized rats, we found only 0.1 cell per penetration with a receptive field or only 1 cell in 10 search tracks. The few receptive fields encountered were on the calf, thigh, lower back, or perineum.

Chronic nerve section. The domination of the medial dorsal horn by toe-foot afferents changed drastically 4 days after peripheral nerve section. From that time on, we found substantial numbers of cells with receptive fields in the medial part of the dorsal horn (Fig. 3), usually extending medially as far as the border of the dorsal column. In 69 search tracks made in 13 rats surviving 4 to 8 days after neurectomy, we found a total of 221 well isolated cells with proximal receptive fields (i.e., a mean of 3.2 cells with receptive field per penetration) and suspect that additional hard-to-isolate units also must have existed. Units were located throughout the dorsal grey matter but mostly in laminae 4 and 5 .

Receptive fields in the medial dorsal horn in intact rats are all located on the toes and/or foot. They respond to stimuli of a single modality, or more commonly, a combination of modalities, and have receptive field size and structure as has been described previously (e.g., Wall et al., 1967; Menetrey et al., 1977; see Fig. 2 for examples). Receptive fields in the medial dorsal horn in chronic neurectomized rats all were located proximal to the ankle with a large preponderance on the upper medial thigh, within the distribution of the posterior cutaneous nerve of the thigh (also called posterior femoral cutaneous 


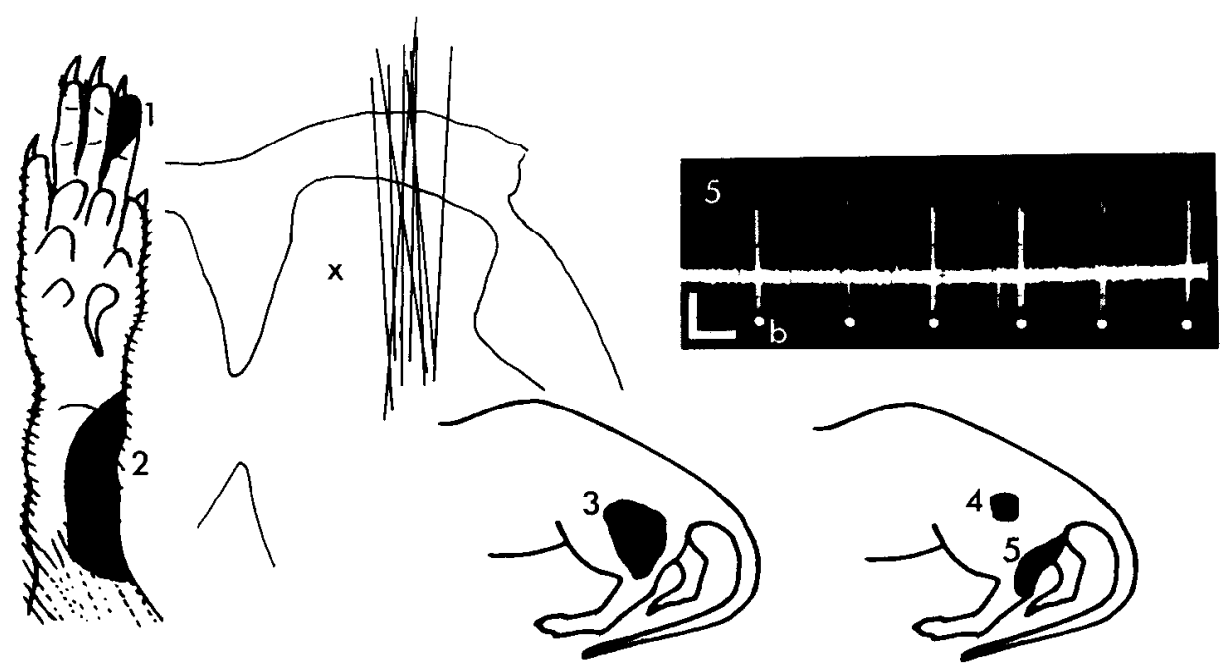

Figure 2. Rearrangement of receptive fields of cells in the medial part of the dorsal horn in rats following transection of the sciatic and saphenous nerves. Vertical electrode penetrations proceeded from the dorsal column laterally into the dorsal horn. The position of the first penetration in which proximal receptive fields occurred in 9 acutely neurectomized rats is indicated by vertical lines on the spinal cord section. The zone medial to these lines was the focus of this study. Black patches 1 and 2 show the size and location of typical receptive fields of medial dorsal horn cells in L4 and 5 in intact rats. Receptive fields 3,4 , and 5 are from the same zone but in a rat that underwent hindlimb neurectomy 4 days earlier. The inset record shows the response of cell 5 to brisk movements of guard hairs within the receptive field (white dots). Scale, $400 \mu \mathrm{V}$; $200 \mathrm{msec}$. 'The position of this cell in the dorsal horn is marked with an $X$ on the spinal cord section.

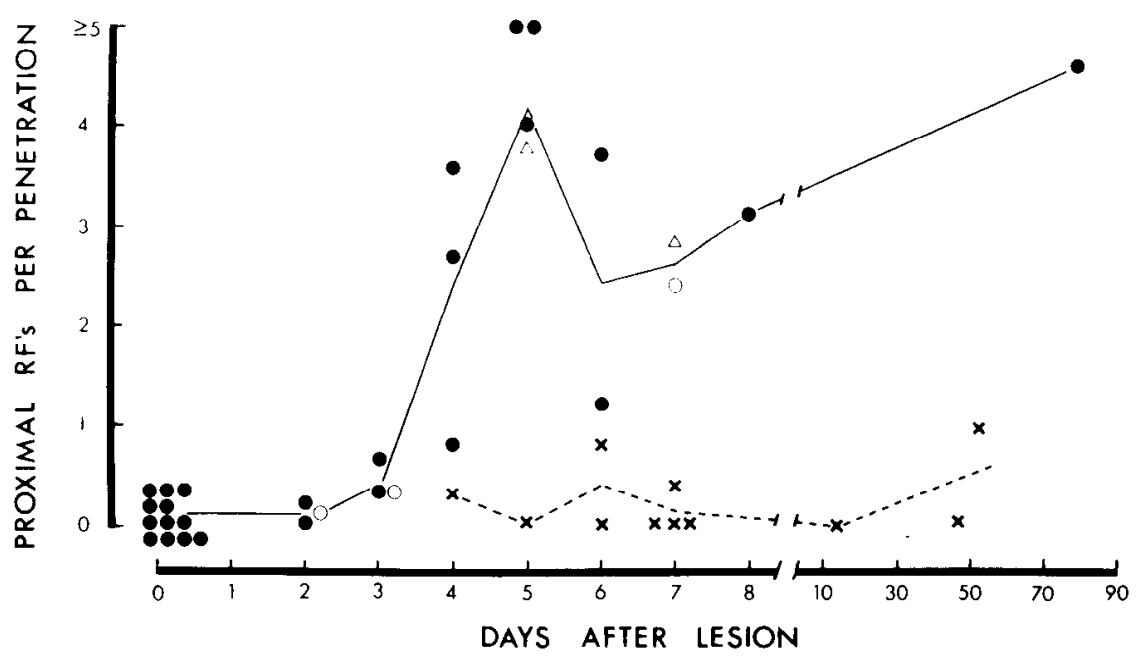

Figure 3. The mean incidence of cells with proximal receptive fields $\left(R F^{\prime \prime} s\right)$ in the medial L4 and 5 dorsal horn rose sharply between the 3rd and 4th days following transection of the sciatic and saphenous nerves (solid line) but not following nerve crush (dashed line). Each symbol indicates data from a single dorsal horn. Many of the data points on day 0 (acute nerve cut) were taken from the control side of chronic experimental rats. Sciatic and saphenous nerves ligated and cut distally; $O$, both nerves cut but not ligated; $\triangle$, both nerves ligated but not cut; $\times$, sciatic nerve crushed and saphenous nerve cut and ligated.

nerve). Transection of this or other nerves of the thigh and perineum invariably eliminated the novel receptive fields. Receptive field sizes ranged from about 0.5 to 15 $\mathrm{cm}^{2}$. Thus, in both size and location, these novel medial dorsal horn receptive fields were more like receptive fields in the lateral part of the dorsal horn of intact rats than the much smaller receptive fields that normally occur in medial dorsal horn (Fig. 2). Adequate stimuli spanned the normal range from small movements of individual hairs (e.g., Fig. 2, cell 5) to firm pinching. Dynamic range was often broad with cells responding to light touch, for example, and increasing their discharge 
as stimulus strength increased. In the rats that survived 4 and 5 days after neurectomy, $30 \%$ of the cells with receptive fields responded to hair movements or light touch; the remainder required firm pressure or pinch. In the rats that survived 6 days or more, $60 \%$ of the cells responded to hair movement or touch. In intact rats, we found that between 80 and $90 \%$ of cells respond to low threshold stimuli (also see Wall et al., 1967; Menetrey et al., 1977). Most proximal receptive field cells in normal rats show a gradation in threshold from relatively low in the receptive field center to relatively high in the periphery. Such structured receptive fields were relatively rare in the present sample of cells, with most showing homogeneous sensitivity throughout the receptive field. Nonetheless, the units were clearly distinguished from primary afferents which have smaller receptive fields and a much narrower range of adequate stimuli. Other indicators that we were recording from cells and not fibers include the general failure to see criterion spikes as the electrode penetrated the dorsal column and the frequent occurrence of characteristic injury discharge as cells were impaled by the micropipette tip. Zones in which mechanical stimulation produced inhibition did occur in these cells, but inhibition was less common than in intact rats. Similar peculiarities were seen previously in the cat (Devor and Wall, 1978, 1981).

Chronic nerve crush. We were interested to learn whether the reorganization of the dorsal horn sensory map that we have described is reversible upon regeneration of the peripheral nerve. From earlier studies (Devor et al., 1979), we know that cutaneous reinnervation of the foot begins about 20 to 25 days after a standard crush of the sciatic nerve in Sabra strain rats. We therefore examined receptive fields in the dorsal horn of two groups of rats with chronic nerve crush. In the first group (9 rats), the animals were studied 4 to 13 days after the sciatic nerve was crushed. These times were chosen as being long enough to permit spinal map reorganization after nerve section but not long enough to permit regenerating sprouts to reach the periphery. In the second group ( 2 rats), the animals survived 48 and 54 days after nerve crush, sufficient time to allow complete reinnervation of the foot and partial maturation of the regenerating nerve sprouts (Devor and Govrin-Lippmann, 1979). In both groups, the saphenous nerve was ligated and cut on the day of the sciatic nerve surgery. This was done in the experiments on sciatic nerve crush, as in the experiments on sciatic nerve cut, in order to discourage saphenous nerve afferents rather than thigh afferents from coming to dominate cells formerly served by the sciatic nerve. It should be emphasized that our spinal cord recordings were made in the center of the sciatic nerve distribution (medial L4 and 5) and at some distance from the saphenous nerve distribution (medial T13 to L3; Devor and Claman, 1980).

To our great surprise, we found no sign of spinal map reorganization in any of the rats in the first group (see Fig. 3). In 49 penetrations in the medial dorsal horn, only 8 cells were found that had cutaneous receptive fields ( 0.2 cell per penetration). This did not differ significantly from the situation on the acutely cut side of the same rats or from the larger group of rats with an acute nerve cut as described above $(p>0.2)$. The 2 rats that survived long enough to permit regeneration of the sciatic nerve had large numbers of medial dorsal horn cells with toefoot receptive fields. Following acute transection of the regenerated nerve, virtually all of these receptive fields vanished. There remained, on average, only 0.3 cell with proximal receptive fields per search track.

Nerve cut and suture. Since nerve crush did not cause central synaptic reorganization in the first place, the data on rats recovered from nerve crush fail to indicate whether the phenomenon is reversible. For this reason, we checked 3 rats that survived 140,147, and 510 days after bilateral ( 2 rats) or unilateral (1 rat) nerve cut with immediate end-to-end suture. By this time, many sensory fibers had re-established connections in the periphery as indicated by recovery of leg flexion upon pinching of the toes and conduction of a compound action potential across the suture line. Mapping of the medial dorsal horn revealed, as expected, many cells with toe-foot receptive fields. Receptive fields on skin proximal to the ankle, however, were rare. In maps in 5 dorsal horns made after the nerves were (re)cut acutely, we found only 10 cclls with proximal receptive fields in 25 search tracks $(0.4$ cell with receptive field per track). Although this figure is slightly higher than in acutely neurectomized animals, it is far lower $(p<0.001)$ than in rats where regeneration had not occurred yet or was prevented. This indicates that central synaptic reorganization after nerve section is reversible upon nerve regencration.

\section{Discussion}

Our data show that the somatotopic mapping of medial dorsal horn cells in the rat changes within 4 or 5 days of peripheral transection of the afferents that normally dominate them and determine their toe-foot receptive fields. In particular, afferents from nearby intact nerves of the thigh come to drive the cells and provide them with a novel receptive field on the skin proximal to the ankle. This change is largely reversed if the cut nerve regenerates. Reorganization of dorsal horn somatotopy does not occur if the toe-foot nerves are crushed but not cut.

We are certain that novel proximal receptive fields in the medial dorsal horn are not a result of aberrant regeneration of the toe-foot nerves into skin of the thigh or of cross-talk between intact thigh nerve fibers and axotomized toe-foot afferents. Sprouting, especially within only 4 or 5 days, can be ruled out because the cut nerve was tied off with a silk suture. Furthermore, in all experiments, the chronically cut nerve was recut acutely on the day of recording, thus disconnecting any potential sprouts from the spinal cord. With regard to cross-talk, we have recorded from hundreds of $\mathrm{L} 4$ and 5 dorsal root fibers that end in a sciatic nerve neuroma (Devor and Wall, 1976; Govrin-Lippman and Devor, 1978) and have never encountered one that had a cutaneous receptive field of any sort. We can also exclude, on several grounds, the possibility that we were picking up extracellular field potentials from cells actually located in the lateral part of the dorsal horn. Spike amplitudes of cells in the lateral dorsal horn were not abnormally large. Finally, when advancing the recording electrode through the medial 
dorsal horn, we frequently impaled and killed cells from which we had just documented a receptive field on thigh skin. We conclude that we are dealing with some form of synaptic adjustment in the spinal cord proper.

The synaptic reorganization that occurred in our rats was essentially identical to that which we saw previously in cats (Devor and Wall, 1978, 1981). The time course for mature reorganization, however, was much shorter, 4 or 5 days compared to about 4 weeks. This might be simply a species difference. On the other hand, it might be related to the smaller distances that a retrograde signal must traverse in rats than in cats (about $90 \mathrm{~mm}$ compared to about $250 \mathrm{~mm}$ ). Indeed, the time course of a number of retrograde phenomena is dependent on distance (Lieberman, 1974; Grafstein, 1975). The mechanism of synaptic reorganization remains uncertain. In a recent morphological study of the spinal distribution of a major type of thigh nerve afferent after foot nerve transection, we failed to find evidence of medially directed sprouting (Devor and Claman, 1980). This possibility, however, cannot be ruled out completely. Another possibility, for which we have accumulated substantial positive evidence, is that thigh afferents normally form contacts, perhaps monosynaptically (Devor and Wall, 1981), on medial dorsal horn cells with toe-foot receptive fields but that their synapses are relatively ineffective. Transection of the toe-foot nerves somehow releases or strengthens this previously existing but "weak" synaptic channel. The data bearing on these two hypotheses have been discussed elsewhere (Devor and Wall, 1978, 1981).

This leaves us with the matters of what signal brings about the synaptic reorganization, be it sprouting or alteration of synaptic efficacy, and why there is a profound difference between nerve cut and crush. Although, at present, we have no comprehensive answers to these questions, several potential explanations can be ruled out. It is not likely that nerve conduction block in the region of the injury is the relevant signal. We know that impulse traffic in primary afferents does not cease with section. The cut end of the nerve becomes a source of spontaneous discharge of substantial proportions by the 4th postoperative day (Wall and Gutnick, 1974; GovrinLippmann and Devor, 1978). Furthermore, a change in the afferent impulse volley does not account in any obvious way for the difference in effectiveness of nerve cut and crush, both of which are followed by ectopic repetitive discharge. Another obvious but unlikely possibility is that the signal is a byproduct of synaptic degeneration or vacation of postsynaptic space. In contrast to the massive terminal degeneration that occurs in the aftermath of dorsal rhizotomy, we have failed to find evidence of terminal degeneration within the relevant time frame at the light microscopic level using the FinkHeimer procedure (Devor and Wall, 1981; M. Devor, unpublished data) nor did Knyihar and Csillik (1976) see frank degeneration at the ultrastructural level. Furthermore, Knyihar and Csillik (E. Knyihar and B. Csillik, personal communication) saw the same "atrophic" changes with both cut and crush. Perhaps, more convincingly, we have demonstrated that the postsynaptic response of dorsal horn cells to afferent volleys carried in both myelinated and unmyelinated afferents following stimulation of the cut peripheral nerve is normal at 4 and 5 days postoperatively and does not begin to decline until about the 10th postoperative day (Wall and Devor, 1981; Wall et al., 1981). Of course, there are clear biochemical changes in the central terminals of peripherally axotomized primary afferents, and axoplasmic transport from the cell body into both the peripheral and the central axon branch is affected (Karlstrom and Dahlstrom, 1973; Lieberman, 1974; Watson, 1974; Grafstein, 1975; Devor and Claman, 1980). The presence of some new substance, or the absence of some old substance, in the axoplasmic freight could constitute a signal for synaptic rearrangement. Such a hypothesis, of course, requires that changes in transport be different for cut and crush lesions. There is some evidence for this (Karlstrom and Dahlstrom, 1973).

Finally, we should consider possible sources of relevant trophic signals. We can rule out the possibility that blockage of some such material originating in the region of the peripheral axon terminals is of importance. Nerve cut and crush are equally effective in blocking transport across the site of injury. There remains the possibility that the signal is picked up from the site of injury itself and transported retrogradely. There is abundant evidence that a broad spectrum of molecules, both exogenous and endogenous, is picked up at sites of nerve damage and transported centrally (Grafstein, 1975; Fink and Gainer, 1980). The difference between cut and crush injury could depend on differential access of these molecules following damage to the local blood-nerve barrier. Alternatively, since, after crush injury, new sprouts grow rapidly and unhindered, they might extend beyond the point of concentration of the relevant trophic substance before a sufficient amount of it is taken up.

Sensory mapping is a fundamental strategy of central nervous system organization. It has long been assumed that maps are formed by tissue-target interactions during embryogenesis and that the mature pattern is highly stable. This conception is especially common with respect to evolutionarily conservative central nervous system structures, such as the spinal cord. The demonstration of massive and rapid synaptic rearrangement in a first order sensory map in adult mammals forces a revision of our thinking about how sensory maps are formed and maintained.

\section{References}

Basbaum, A. I., and P. D. Wall (1976) Chronic changes in the response of cells in adult cat dorsal horn following partial deafferentation: The appearance of responding cells in a previously non-responsive region. Brain Res. 116: 181-204.

Brown, P. B., and J. L. Fuchs (1975) Somatotopic representation of hind limb skin in cat dorsal horn. J. Neurophysiol. 38: $1-19$.

Devor, M., and D. Claman (1980) Mapping and plasticity of acid phosphatase afferents in the rat dorsal horn. Brain Res. 190: $17-28$.

Devor, M., and R. Govrin-Lippmann (1979) Maturation of axonal sprouts after nerve crush. Exp. Neurol. 64: 260-270.

Devor, M., and P. D. Wall (1976) Type of sensory nerve fibre sprouting to form a neuroma. Nature 262: 705-708.

Devor, M., and P. D. Wall (1978) Reorganization of spinal cord sensory map after peripheral nerve injury. Nature 275: 75 76. 
Devor, M., and P. D. Wall (1981) The effect of peripheral nerve injury on receptive fields of cells in the cat spinal cord. J. Comp. Neurol., in press.

Devor, M., D. Schonfeld, Z. Seltzer, and P. D. Wall (1979) Two modes of cutaneous reinnervation following peripheral nerve injury. J. Comp. Neurol. 185: 211-220.

Fink, D. J., and H. Gainer (1980) Retrograde axonal transport of endogenous proteins in sciatic nerve demonstrated by covalent labeling in vivo. Science 280: 303-305.

Goldberger, M., and M. Murray (1974) Restitution of function and collateral sprouting in cat spinal cord: The deafferented animal. J. Comp. Neurol. 158: 37-54.

Govrin-Lippmann, R., and M. Devor (1978) Ongoing activity in severed nerves: Source and variation with time. Brain Res. 159: 406-410.

Grafstein, B. (1975) The nerve cell body response to axotomy. Exp. Neurol. 48: 32-51.

Illis, L. S. (1973) Experimental model of regeneration in the central nervous system. Brain 96: 47-60.

Karlstrom, L., and A. Dahlstrom (1973) The effect of different types of axonal trauma on the synthesis and transport of amine storage granules in rat sciatic nerves. J. Neurobiol. 4: 191-200.

Knyihar, E., and B. Csillik (1976) Effects of peripheral axotomy on the fine structure and histochemistry of the Rolando substance: Degeneration atrophy of central processes of pseudounipolar cells. Brain Res. 26: 73-87.
Lieberman, A. R. (1974) Some factors affecting retrograde neuronal responses to axonal lesions. In Essays on the Nervous System, R. Bellairs and E. G. Gray, eds., pp. 71-105, Clarendon Press, Oxford.

Liu, C. N., and W. W. Chambers (1958) Intraspinal sprouting of dorsal root axons. Arch. Neurol. Psychiatry 79: 46-61.

Menetrey, D., G. J. Giesler, and J. M. Besson (1977) An analysis of response properties of spinal cord dorsal horn neurones to non-noxious and noxious stimuli in the spinal rat. Exp. Brain Res. 27: 15-33.

Wall, P. D., and M. Devor (1981) The effect of peripheral nerve injury on dorsal root potentials and on transmission of afferent signals into the spinal cord. Brain Res. 209: 95-111.

Wall, P. D., and M. Gutnick (1974) Ongoing activity in peripheral nerves: The physiology and pharmacology of impulses originating from a neuroma. Exp. Neurol. 43: 580-593.

Wall, P. D., and R. Werman (1976) The physiology and anatomy of long-ranging afferent fibers within the spinal cord. J. Physiol. (Lond.) 255: 321-334.

Wall, P. D., J. Freeman, and D. Major (1967) Dorsal horn cells in spinal and in freely moving rats. Exp. Neurol. 19: 519-529.

Wall, P. D., M. Fitzgerald, and S. J. Gibson (1981) The response of rat spinal cord cells to unmyelinated afferents after peripheral nerve section and after changes in substance $\mathrm{P}$ levels. Neuroscience, in press.

Watson, W. E. (1974) Cellular responses to axotomy and related procedures. Br. Med. Bull. 30: 112-115. 